\title{
Posterior based circumferential spinal cord decompression in paediatric patients with the vertebral column resection (VCR) technique spares the anterior approach in severe kyphosis
}

\author{
Dunn RN ${ }^{1} \mathbb{D}$, Horn $\mathrm{A}^{2} \mathbb{C}$
}

1 1BChB(UCT), FCS(SA)Orth, MMed (Orth)(UCT); Consultant Spine and Orthopaedic Surgeon, Pieter Moll and Nuffield Chair of Orthopaedic Surgery, Department of Orthopaedic Surgery, University of Cape Town, South Africa

2 MBChB, FC Orth (SA), MMed(Orth)(UCT); Consultant, Red Cross Children's Hospital, Department of Orthopaedic Surgery, University of Cape Town, South Africa

Corresponding author: Prof RN Dunn, Dept of Orthopaedic Surgery, H49 Old Main Building, Groote Schuur Hospital, Anzio Road, Observatory, Cape Town; cell: 021404 5118; email: robert.dunn@uct.ac.za

\begin{abstract}
Introduction: Patients with kyphosis and myelopathy have traditionally been managed by combined anterior and posterior surgery with associated morbidity and long theatre episodes. The posterior vertebral column resection (pVCR) technique allows circumferential apical decompression with instrumented correction and stabilisation through a single extra-pulmonary approach. The objective of this study was to review the use of pVCR in the paediatric population as to feasibility, safety and effectiveness.

Methods: A retrospective review of prospectively maintained database was undertaken. Twenty-six consecutive paediatric patients (18 females) were reviewed. The median age at surgery was 12.1 years (IQR 7.6-14.2, range 2.1-17.7). The underlying pathology was active tuberculosis in nine patients, healed tuberculosis in seven, congenital in seven, tumour in two and trauma in one. Myelopathy was present in 17 and two had associated syringomyelia. The median surgical duration was 3.3 hours (IQR 3-5.0, range 1.9-6.5) with a blood loss of $20.8 \mathrm{ml} / \mathrm{kg}$ (IQR 12.5-38.1, range 6.3-67.6).

Results: The median percentage sagittal correction was 65\% (IQR 44-79, range 21-100). The correction was best in the thoracolumbar spine at $75 \%$. All non-ambulatory patients improved to an ambulatory status. There was one permanent and one transient neurological deterioration. All intact patients (ASIA E) remained so. One of the two patients that had associated syringomyelia had complete resolution of the syrinx with improved neurological function. There were no related pulmonary complications. One patient developed early (in-hospital) proximal junctional failure requiring revision.

Conclusion: Posterior based circumferential decompression and corrective fusion using the pVCR technique is both feasible and effective in the paediatric population. It avoids the morbidity associated with the trans-thoracic approach and allows improvement both in neurological function and CSF dynamics when they are compromised.

It remains a technically challenging procedure both for the surgical and anaesthetic team and there needs to be a clear understanding of the risk-benefit relationship when deciding on its implementation.
\end{abstract}

Level of evidence: Level 4

Key words: vertebral column resection, VCR, myelopathy, kyphosis, paediatric

Citation: Dunn RN, Horn A. Posterior based circumferential spinal cord decompression in paediatric patients with the vertebral column resection (VCR) technique spares the anterior approach in severe kyphosis. SA Orthop J 2019;18(2):20-24. http://dx.doi.org/10.17159/2309-8309/2019/ v18n2a1

Editor: Prof LC Marais, University of KwaZulu-Natal, Durban, South Africa

Received: September 2018

Accepted: February 2019

Published: May 2019

Copyright: ( 2019 Dunn RN, Horn A. This is an open-access article distributed under the terms of the Creative Commons Attribution Licence, which permits unrestricted use, distribution and reproduction in any medium, provided the original author and source are credited.

Funding: No funding was received for this study.

Conflict of interest: The authors have no conflicts of interest with respect to this study. 


\section{Introduction}

The management of paediatric patients with severe rigid spinal deformity, particularly with associated spinal cord compression and myelopathy, is extremely challenging due to the risk of neurological injury and the difficult internal fixation of their small, distorted spines. Traditionally pre-operative traction has been employed to reduce the deformity, but due to their low body weight this is frequently ineffective. Although anterior transthoracic release and decompression has been utilised, gaining access to the apex of the deformity to relieve cord compression can be technically difficult. Typically partial deformity correction with anterior column strut grafts have been employed.

A posterior approach allows the surgeon to place segmental instrumentation which is powerful enough to obtain and maintain correction, while fusion occurs. This is then combined with an anterior decompression/release, either simultaneously or in a staged fashion. This strategy's dual surgical approach comes at the cost of time and large physiological insult.

With the development of the posterior based circumferential vertebral column resection ( $\mathrm{pVCR}$ ), the deformity can be well visualised. Rigid posterior fixation and visualisation of the thecal sac by laminectomy allows better orientation and more controlled removal of bone anterior to the cord than the anterior approach. ${ }^{2-4}$

We have increasingly employed the pVCR technique in our paediatric patients that present with severe rigid deformity with established or predicted myelopathy.

We present our experience with this technique in the paediatric group in an effort to assess feasibility, safety and effectiveness.

\section{Methods}

Following institutional ethics approval, we identified 26 paediatric patients ( $\leq 18$ years old) undergoing the pVCR procedure from January 2010 to April 2017 from a prospectively maintained database. A case note and imaging review were conducted with regard to demographics, surgical indications and complications. Outcome was assessed radiographically in terms of deformity correction and clinically in terms of neurological status.

Data was captured and analysed on Microsoft Excel spreadsheets. Due to the relatively small numbers, median and inter-quartile ranges (IQRs)were used to avoid the distortion effect of outliers on average and ranges.

Our cohort had a median age of 12.1 years (IQR 7.6-14.2, range 2.1-17.7). There was a female predominance of 18 to 8 (Figure 1 ).

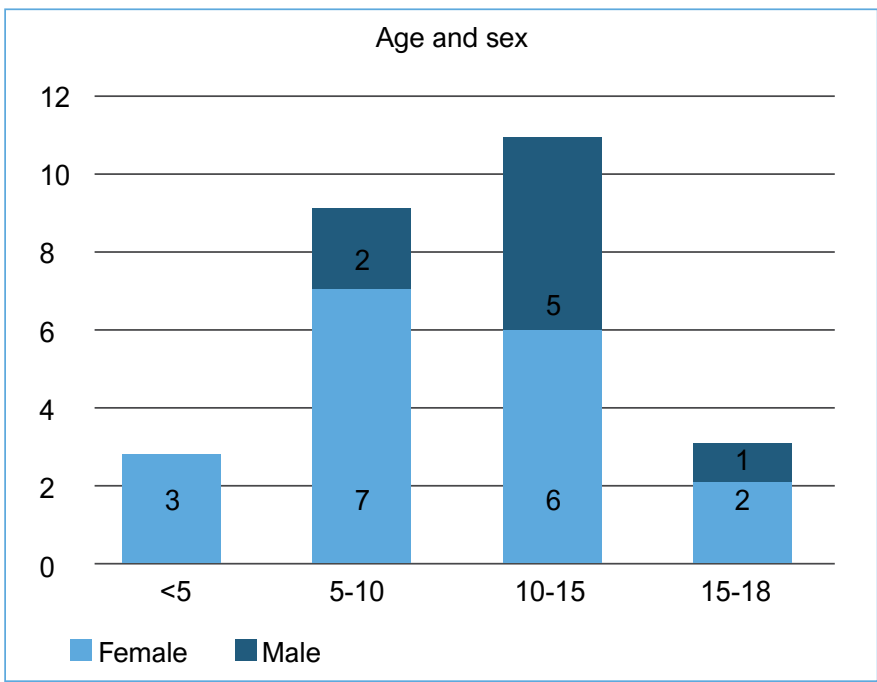

Figure 1. Patient age distribution with female predominance (blue)

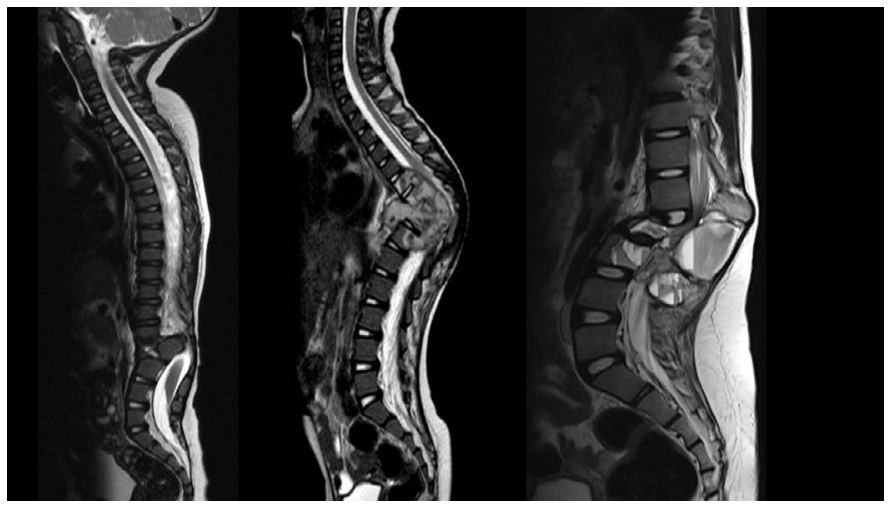

Figure 2. Pre-operative MRIs confirming congenital spine dislocation, active tuberculosis and aneurysmal bone cyst ( $L$ to $R$ ) as causes of kyphosis

The commonest underlying pathology was tuberculosis, both active (nine cases) and healed disease (seven) followed by congenital anomalies (seven) (Table I) (Figure 2).

The indication for the procedure was kyphotic deformity, frequently associated with myelopathy. Two cases had associated syringomyelia. The commonest site was the thoracic spine (14 patients) followed by thoracolumbar and lumbar spine (six patients each).

\section{The procedure}

Patients were positioned prone under total intravenous anaesthesia when spinal cord monitoring (SCM) was used. In the earlier part of the study SCM was not available but later, when neurological status allowed, surgeon-operated transcranial motor-evoked monitoring was performed. This is now considered routine practice by the authors. $^{5}$

The spine was exposed and segmental pedicle screw instrumentation placed. Fixed head screws were usually used but in the smaller children an adult cervical instrumentation system with polyaxial screws was utilised. Once the surgeon was satisfied that rigid fixation was achieved, the pVCR was commenced.

The thecal sac was exposed with laminectomy of the planned pVCR site. Bilateral costotransversectomies were performed, resecting as many ribs as required to access the anterior column. An upcut (typically $5 \mathrm{~mm}$ ) was used to follow the rib down to the side of the vertebral body. This plane was developed with blunt dissection (cottonoid on a Kocher clamp) to visualise the vertebral discs and bodies, placing a copper malleable retractor to maintain this space, typically clipped to the drapes. Thoracic roots were sacrificed when access required it, often more on one side to allow subsequent graft placement. The roots were tied distal to the dorsal root ganglion with $2 / 0$ Vicryl and cut with a blade. The sutures were kept long and clipped to lift and retract the dural sac and allow access to the posterior body wall. The discs and vertebral bodies were resected with curettes, upcuts and bone-nibblers, preserving

Table I: Underlying pathology

\begin{tabular}{|c|c|}
\hline Congenital & 7 \\
\hline Failure of formation & 4 \\
\hline Dislocations & 3 \\
\hline Incomplete SCI & 1 \\
\hline Tuberculosis & 16 \\
\hline Active & 9 \\
\hline Healed & 7 \\
\hline Tumour & 2 \\
\hline
\end{tabular}


Table II: Sagittal correction

\begin{tabular}{|c|c|c|c|c|c|c|c|c|}
\hline & Pre-op & & Post-op & & Change & & $\%$ change & \\
\hline Lumbar & $35.5^{\circ}$ & $\begin{array}{c}\text { IQR } 26-40.5 \\
16-47\end{array}$ & $6^{\circ}$ & $\begin{array}{c}\text { IQR 1-17 } \\
0-34\end{array}$ & $18^{\circ}$ & $\begin{array}{c}\text { IQR 9-37 } \\
2-47\end{array}$ & $66 \%$ & $\begin{array}{l}\text { IQR 44-100 } \\
6-100\end{array}$ \\
\hline Thoracic & $64^{\circ}$ & $\begin{array}{l}\text { IQR 55-88 } \\
30-100\end{array}$ & $27^{\circ}$ & $\begin{array}{c}\text { IQR } 17-30 \\
8-70\end{array}$ & $28^{\circ}$ & $\begin{array}{l}\text { IQR 20-64 } \\
\text { प12-71 }\end{array}$ & $55 \%$ & $\begin{array}{l}\text { IQR 40-73 } \\
\text { प21-87 }\end{array}$ \\
\hline TL & $90^{\circ}$ & $\begin{array}{c}\text { IQR } 77-96 \\
46-99\end{array}$ & $22^{\circ}$ & $\begin{array}{c}\text { IQR } 19-46 \\
0-65\end{array}$ & $49^{\circ}$ & $\begin{array}{c}\text { IQR } 44-62 \\
30-80\end{array}$ & $73 \%$ & $\begin{array}{c}\text { IQR } 51-80 \\
32-100\end{array}$ \\
\hline
\end{tabular}

Table III: Neurological status

\begin{tabular}{|c|c|c|c|c|c|c|c|}
\hline & & \multicolumn{6}{|c|}{ Last follow-up } \\
\hline \multirow{6}{*}{$\begin{array}{l}\frac{5}{0} \\
\frac{0}{8} \\
\frac{0}{8}\end{array}$} & & & A & B & C & D & $E$ \\
\hline & A & 0 & & & & & \\
\hline & B & 0 & & & & & \\
\hline & C & 9 & & & & 3 & 6 \\
\hline & D & 8 & 1 & & & 2 & 5 \\
\hline & $E$ & 9 & & & & & 9 \\
\hline
\end{tabular}

the posterior body wall until late. A plane between the theca and posterior longitudinal ligament is developed before this posterior wall is finally broken into the cavity created. Care should be taken to avoid an anterior durotomy as it can be challenging to repair. During this process a temporary rod was placed to stabilise the spine and prevent cord injury.

Once the circumferential decompression was complete, the screws holding the temporary rod were loosened allowing some in situ rod bending while avoiding distraction with increased cord tension as the rod could slide through the screw heads. An anterior strut graft was placed and definitive rods sequentially applied with further sagittal correction and compression across the graft. In cases, especially the earlier ones, when struts could not be placed or were deemed unnecessary, morcellised graft was placed anteriorly. The remaining allograft strut was cut into bone plates and placed from the remaining cephalad to the caudal laminas to cover the theca. They were usually sutured in through drill holes to avoid migration. The wound was closed over a drain.

Allograft fibula shafts were used in 19 cases, a prosthetic cage in one and morcellised graft in six.

The median duration of surgery (skin to skin) was 3.3 hours (IQR 3-5.0, range 1.9-6.5).

The median blood loss was $500 \mathrm{ml}$ (IQR 400-1225, range 100-2500). This represents a median $20.8 \mathrm{ml} / \mathrm{kg}$ (IQR 12.5-38.1, range 6.3-67.6).) Cell-saving was only used in the larger children.

Spinal cord monitoring was used in 13 cases, with three cases failing to obtain signals. In the ten cases monitored there were two alerts while correcting the deformity which required remedial action.

The median follow-up was 16 months (IQR 10.5-33.9).

\section{Results}

The median overall percentage sagittal correction was 65\% (IQR 44-79, range 21-100). The correction was best in the thoracolumbar spine at $75 \%$, followed by the lumbar spine (Table II).

There was dramatic improvement in the neurological status as presented in Table III. All non-ambulatory patients (ASIA C) improved to an ambulatory status. Of the ASIA Ds (walking but not normal power) most were normal at follow-up, two were unchanged at short-term follow-up, and one deteriorated. All neurologically normal patients (ASIA E) remained so.
There was one permanent neurological deterioration from ASIA $D$ to $A$ in a patient with a congenital $L 1 / 2$ dislocation and tethered cord. A short instrumentation was done to minimise growth disturbance as she was only 2 years old. The patient returned 12 weeks post-operatively with fixation failure and catastrophic neurological deterioration. Despite revision, she remained an ASIA A (Figure 3).

One patient had a transient neurological deterioration postoperatively. On re-MRI, incomplete decompression was confirmed with ongoing cord compression from the postero-inferior edge of the superior vertebral body. This was revised a week later with subsequent neurological recovery.

One of the two patients that had associated syringomyelia had complete resolution of the syrinx on follow-up MRI with improved neurological function (Figure 4).

One patient developed early (in-hospital) proximal junctional failure. Following an excellent correction, she developed a proximal facet dislocation requiring cephalad extension of her instrumentation. There were no neurological sequelae, however (Figure 5).

One case developed surgical site sepsis requiring washout and subsequent instrumentation removal.

There were no pulmonary complications.

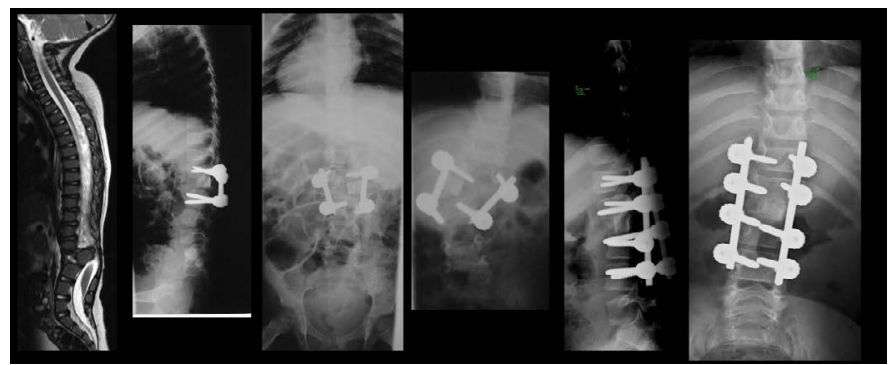

Figure 3. Two-year-old patient with congenital spine dislocation who presented 12 weeks post-surgery with paraplegia and failed correction due to inadequate instrumentation - kept short to allow spine growth. Despite revision she did not recover neurologically as despite lumbar, it was a cord level injury due to her tethered cord and low conus.

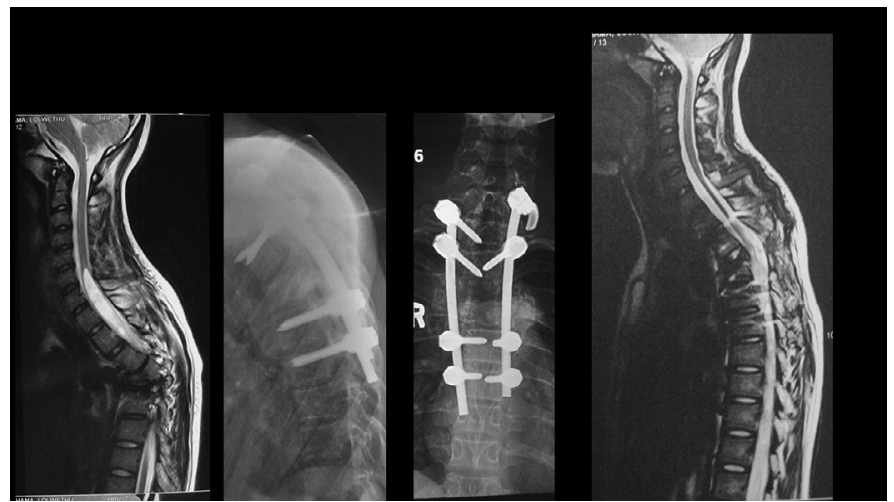

Figure 4. Pre-operative syrinx totally resolved on follow-up scan after VCR 


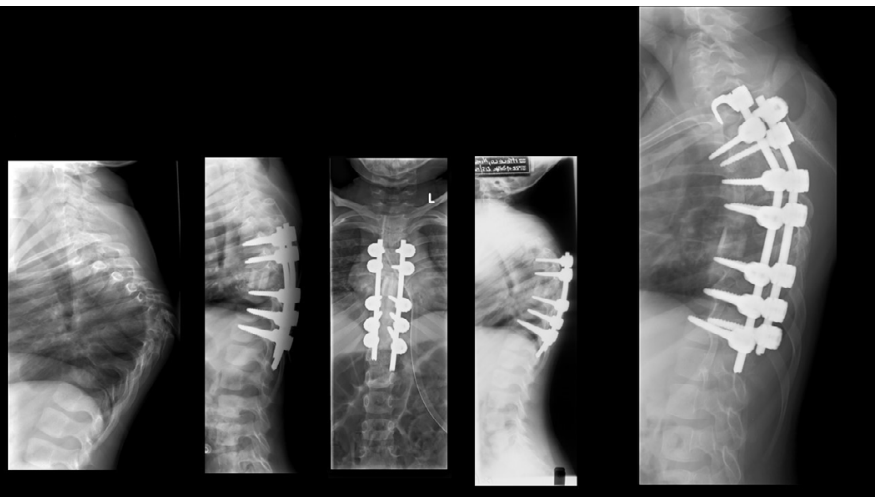

Figure 5. Following excellent sagittal plane correction, this young child developed early proximal junctional failure while still in hospital requiring cephalad extension of the construct. There were no neurological sequelae, however.

\section{Discussion}

The pVCR was originally described by Suk et al. for correction of severe deformity in an adult population. ${ }^{3}$ Their focus, as with other authors, was largely around deformity correction., ${ }^{4,6-8}$ With the risk of neurological deterioration we are hesitant to promote such aggressive methods for deformity alone, but feel it is indicated when cord compression is present with established or predicted myelopathy.

South Africa has the rare combination of developing world pathology of advanced disease processes and developed world care. This is apparent in our cohort where paediatric patients present late with severe kyphotic deformity, many with established or impending myelopathy. It is tempting to defer aggressive management until a significant neurological deficit is present to justify the risk of intervention, but the more advanced the myelopathy, the less recovery can be expected. This and dissatisfaction with traditional anterior and posterior surgery has led us to steadily increase our use of the pVCR in this group. ${ }^{1}$

Although technically challenging, it allows circumferential decompression of the spinal cord with excellent visualisation and correction of angular deformity. In addition, it allows spinal column shortening, thereby reducing tension on the cord and the risk of neurological injury.

Our experience confirms that pVCR is technically possible in a paediatric population of all ages. We successfully decompressed all patients with neurological improvement in the vast majority. All patients went on to a stable fusion with no rod breakage.

Instrumentation failures were the result of including too few levels in the fusion, compromising stability to allow more spinal growth in the future. The worst complication was the result of a short fixation in a 2-year-old. The other was a proximal failure in a severe kyphosis with massive correction, requiring proximal extension of the construct.

The circumferential approach allows control of the spine at all times as compared to the more traditional anterior decompression and posterior fusion where transthoracic decompression and strut placement was followed by re-positioning and posterior surgery with risk of graft displacement in the process.

In addition, the correction is performed in a controlled manner with visualisation of the thecal sac where the tension can be confirmed and avoided.

The procedure is demanding with prolonged operating times and large blood loss reported. Suk reported an average of 4.5 hours operating time and $2300 \mathrm{ml}$ blood loss in adults. Lenke in his series of 147 paediatric cases reported just under 10 hours operating time and $1610 \mathrm{ml}$ blood loss, representing $65 \%$ of blood volume. Our series compares favourably at 3.3 hours and $500 \mathrm{ml}$ representing $30 \%$ of blood volume. The case mix is somewhat different with ours being largely sagittal plane deformity as opposed to scoliosis. In addition, the senior author performed all the surgery in our cohort whereas the Lenke series had seven surgeons. Our use of freehand pedicle screw insertion with fluoroscopy only used to confirm the level may also contribute to reduced surgical duration. Paradoxically, despite the complexity, the smaller the patient, the quicker the procedure as there is far less to resect.

Complication rates are high, with Lenke reporting a 59\% incidence. Suk had two paraplegias in his 70 cases whereas Lenke had $27 \%$ intra-operative SCM events but no permanent paraplegia. In our series there was one transient deterioration and one catastrophic deterioration 3 months post-operatively related to instrumentation failure. We had a $20 \%$ SCM alert incidence which allowed intra-operative intervention to relieve stress on the cord. This risk makes SCM mandatory for this type of procedure.

We achieved excellent sagittal correction with this technique, comparable with the literature..$^{3,48}$ It was slightly less in the thoracic spine compared to the lumbar and thoracolumbar. This is probably due to the anterior tethering effect of the costosternal joint complex. One needs to be cognisant of the forces generated when correcting the deformity as our only proximal failure was in a massive correction in a very young patient.

No pulmonary complications were encountered as the pleural cavity is not violated. The reduced pulmonary function following transthoracic approaches is well documented, and avoiding this with the pVCR technique may make it safer in these young children with chest deformity and probably reduced respiratory reserve. ${ }^{9}$

\section{Conclusion}

Posterior based circumferential decompression and corrective fusion using the pVCR technique is both feasible and effective in the paediatric population. It avoids the morbidity associated with the trans-thoracic approach and provides improvement in kyphosis, neurological function and CSF dynamics when they are compromised.

It remains a technically challenging procedure both for the surgical and anaesthetic team and there needs to be a clear understanding of the risk-benefit relationship when deciding on its implementation.

\section{Ethics statement}

All procedures followed were in accordance with the ethical standards of the responsible committee on human experimentation (institutional and national) and with the Helsinki Declaration of 1975 , as revised in 2008 . Following database interrogation all data was anonymised.

\section{Declaration}

The authors declare authorship of this article and that they have followed sound scientific research practice. This research is original and does not transgress plagiarism policies.

\section{Author contributions}

RND maintained the database, performed the surgery, assisted with preparation of the manuscript and performed the revision.

$\mathrm{AH}$ collated the data, performed the primary analysis and assisted with preparation of the manuscript. 


\section{ORCiD}

RN Dunn (iD http://orcid.org/0000-0002-3689-0346

A Horn (iD http://orcid.org/0000-0002-4159-6520

\section{References}

1. Dunn R, Garrett B. Surgery for myelopathy in spinal deformity. $S A$ Orthopaedic Journal 2011;10(1):30-34.

2. Abdullah M, Enrico N, Dunn R. Posterior vertebral column resection for severe spinal deformities. SA Orthopaedic Journal 2012;11(4):21-25.

3. Suk SI, Kim JH, Kim WJ, Lee SM, Chung ER, Nah KH. Posterior vertebral column resection for severe spinal deformities. Spine 2002;27(21):2374-82.

4. Lenke LG, Newton PO, Sucato DJ, Shufflebarger HL, Emans JB, Sponseller PD, et al. Complications after 147 consecutive vertebral column resections for severe pediatric spinal deformity: a multicenter analysis. Spine 2013;38(2):119-32.

5. Dunn R. Surgeon operated trans-cranial motor evoked potentials (tcMEP) in spinal deformity surgery - a viable option in resourced challenged environments? IMAST abstract 64 Cape Town 2017. p 84.

6. Mattila M, Jalanko T, Helenius I. En bloc vertebral column derotation provides spinal derotation but no additional effect on thoracic rib hump correction as compared with no derotation in adolescents undergoing surgery for idiopathic scoliosis with total pedicle screw instrumentation. Spine 2013;38(18):1576-83.

7. Lu G, Wang B, Li Y, Li L, Zhang H, Cheng I. Posterior vertebral column resection and intraoperative manual traction to correct severe post-tubercular rigid spinal deformities incurred during childhood: minimum 2-year follow-up. Eur Spine $J$ 2015;24(3):586-93.

8. Hamzaoglu A, Alanay A, Ozturk C, Sarier M, Karadereler S, Ganiyusufoglu K. Posterior vertebral column resection in severe spinal deformities: a total of 102 cases. Spine 2011;36(5):E340-44.

9. Redding GJ. Early onset scoliosis: a pulmonary perspective. Spine Deform 2014;2(6):425-29. 